\section{On nonlinear horizontal dynamics and vibrations control for high-speed elevators}

\author{
DR Santo', JM Balthazar ${ }^{1,2}$, AM Tusset ${ }^{3}, V$ Piccirilo $^{3}$, \\ RMLRF Brasil ${ }^{4}$ and M Silveira'
}

2018, Vol. 24(5) 825-838

(C) The Author(s) 2016

Reprints and permissions: sagepub.co.uk/journalsPermissions.nav DOI: 10.1 I 177/1077546316667324 journals.sagepub.com/home/jvc

SAGE

\begin{abstract}
In this work, the horizontal nonlinear response of a three-degree-of-freedom vertical transportation model excited by guide rail deformations is investigated. The equation of motion contains nonlinearities in the form of Duffing stiffness for the translational spring in tilting motion of the cabin. In order to improve the comfort for passengers a control strategy based on the State-dependent Ricatti Equation (SDRE) is proposed. Numerical simulations are performed to study the nonlinear behavior of the adopted mathematical model. In addition, we test the robustness of the SDRE control technique considering parametric errors and noise. The obtained results confirm that the proposed strategy can be effective in controlling the response of the system.
\end{abstract}

\title{
Keywords
}

Mathematical modeling, vertical transportation, horizontal vibrations, chaos, State-dependent Ricatti Equation control

\section{Introduction}

The exponential growth of urban centers has forced cities to develop themselves predominantly in the vertical direction. This development is achieved by means of advancement in the technology of construction of towers and very tall buildings. To supply the demand for these constructions, the development of high-speed elevators has become a necessity. As an example, in Taipei, Taiwan, the Taipei 101 building is $508 \mathrm{~m}$ tall and has two elevators working at speeds in excess of $60 \mathrm{~km} / \mathrm{h}$ (Munakata et al., 2003). However, high speeds can result in decreased quality of ride (Mitsui and Nara, 1971), this being one of the main problems in high-speed elevators systems (Nai et al., 1994). Thus, it is necessary at the same time to increase the speed limit and improve the travel quality, without losing the efficiency of elevators. Although there is no speed limit that passengers can tolerate, there are limits on other parameters, such as levels of horizontal and vertical vibrations, lateral and longitudinal acceleration and jerk, all of which are necessary to ensure good ride quality for passengers (Fortune, 1997).

According to Roberts (1998), efficiency is related to how fast people move within buildings, the level of energy required to achieve these transitions and the volume used by the transportation system inside the building. In high-speed passenger elevators, the safety and comfort of the passengers is essential. Therefore, to provide a comfortable environment for passengers is a priority for skyscraper elevators. Three factors define passenger comfort: noise pollution/disturbance; atmospheric pressure inside the cabin; and vertical and horizontal vibrations levels, which, in addition to efficiency, guarantee good ride quality.

In the current literature, it is possible to find different models for elevator systems. Research on these systems generally focuses on vertical and horizontal vibration levels inside the cabin of the elevator, aiming at a better understanding of elevator dynamics and, therefore, improving the ride quality offered to passengers.

\footnotetext{
'Department of Mechanical Engineering, UNESP-Bauru, SP, Brazil ${ }^{2}$ Mechanical-Aeronautics Division, ITA-Sao Jose dos Campus, SP, Brazil ${ }^{3}$ Department of Mathematics, UTFPR-Ponta Grossa, PR, Brazil ${ }^{4}$ Center for Engineering, Modeling and Applied Social Sciences, UFABCSanto Andre, SP, Brazil
}

Received: I March 2016; accepted: II August 2016

\section{Corresponding author:}

AM Tusset, Department of Mathematics, UTFPR-Ponta Grossa, PR, Av. Monteiro Lobato, Km 04, s/n. CEP: 20-840 I6-2 10 - Ponta Grossa-PR, Brazil.

Email: a.m.tusset@gmail.com 
Chi and Shu (1991) studied vertical vibrations of elevator systems under harmonic excitation. Zhu and Teppo (2003) built a scale model to simulate lateral linear vibrations in a traction cable, with varying length, used in high-speed elevators. Taking into account the length of hoisting cables and compensating ropes, the nonstationary dynamic behavior of an elevator system and cabin vibrations due to guide rail stochastic excitations, unevenness and imperfections were investigated (Kaczmarczyk and Iwankiewicz, 2006; Kaczmarczyk et al., 2009). Other works on elevator system dynamics and their components have been carried out by several authors (Fu et al., 2005; Zhang et al., 2008).

Lateral vibrations of the cabin during elevator operation are one of the most important factors that can affect the comfort of the passengers (Noguchi et al., 2011). Currently, with increasing building height and elevator speed, these vibrations reach large amplitudes due to excitations caused by misalignments and deformations of guide rails and air pressure disturbance around the cabin. To improve the comfort of the passengers, elevators should have a good control system, in order to increase the positioning accuracy and to guarantee that the speed of the elevator does not cause discomfort to the passengers, by decreasing the vertical and horizontal vibrations of the elevator cabin.

A number of works are found in the literature that attempt to suppress undesirable vibrations in elevator systems. Kaczmarczyk and Picton (2013) used a traction drive elevator system with long ropes and cables in order to analyze high-rise structures. A multi-modal active stiffness controller is proposed, which substantially reduced the response and the effects of passage through primary resonances. Benosman (2014) studied numerically the problem of elevator rope with sway motion due to external force disturbances through an active control, by using nonlinear controllers based on the Lyapunov theory, to stabilize the rope sway dynamics. In Sandilo and van Horssen (2015), a model for the transversal vibrations of an elevator cable system is studied. The initial-boundary value problem for a linear axially moving string equation with time varying length, space-time-varying tension and a constant velocity is studied. The upper end of the string is excited sinusoidally, whereas the lower end of the string is assumed to be fixed. An active suspension system with electromagnetic actuators was used to suppress vibration in the elevator car (Utsunomiya et al., 2004, 2006). A robust controller was designed to reduce horizontal vibrations of highspeed elevators using the Lyapunov method by Feng et al. (2009). Funai et al. (2004) showed a comparison between two active vibration dampers for super highspeed elevators. The first strategy uses active roller guides, and it is observed that this procedure is effective for elevators that are affected, mainly, by guide rail deformation. The second technique is an electromagnetic actuator installed between the car frame and platform, and this is more appropriate for elevators that are greatly affected by air pressure disturbance. Lopez et al. (2010) proposed the development of an adaptive control for active suspension of high-performance elevators, based on the pole-placement method, augmented with a self-tuning scheme for compensating the variations in the mass of the system as the number of passengers changes with time. The authors demonstrated that adaptive pole-placement can reduce the lateral vibrations in the base of the cabin and its efficiency is better than other control strategies, such as proportional-integral-derivative (PID), Sky Hook and pole-placement. In Chang et al. (2011), a high-speed elevator system was investigated in order to examine the characteristics of the excitations and analyze the dynamic responses due to the horizontal vibration generated from the elevator wheels running on rough and winding guide rails. An active mass driver based on the $\mathrm{H} \infty$ direct output feedback control algorithm was utilized to reduce the horizontal acceleration of the passenger car in the elevator. In Yang et al. (2014), an active control with time variant states using the Co-FXLMS (Correlation Filtered-X Least Mean Square) algorithm and MBPF (Moving Band Pass Filter) was proposed to control the cabin noise of the high-speed elevator. Zhang et al. (2014) obtained the exact value of fault probability of components in the elevator system by combining fuzzy theory with the Bayesian network approach. In addition is proposed a reliability analysis method of the multi-state system based on fuzzy Bayesian networks; the results provide a quantitative evaluation for the reliability of multi-state horizontal vibration of the elevator. Arrasate et al. (2014) conducted a study of vertical vibrations caused by torque ripple generated at the elevator drive system and its influence on passenger comfort during elevator travel. Experimental tests have been performed to study vertical vibrations of the car and counterweight assembly due to excitations generated at the drive system in an elevator installation. Venkatesh et al. (2002) presented a methodology for designing high performance of the LTI (linear time-invariant) controller by elevator vertical motion for high-rise buildings with high speed. Simulations show that the controller improves the vertical ride quality and is insensitive to parametric changes that typically occur as a result of normal wear and tear.

The State-dependent Ricatti Equation (SDRE) strategy is an interesting algorithm for synthesizing nonlinear feedback controls. It allows for nonlinearities in the system states while additionally offering great design flexibility through state-dependent weighting matrices (Çimen, 2010). It is an effective algorithm to 
suppress undesirable dynamic behaviors. It was first proposed by Pearson (1962), and has been applied in a wide variety of nonlinear control applications, such as autopilot design (Mracek, 2007), satellite and spacecraft control (Stansbery and Cloutier, 2000), micro electromechanical systems (MEMS) with chaotic behavior (Tusset et al., 2012a, 2013), Atomic Force Microscopy (AFM) systems with chaotic behavior (Nozaki et al., 2013; Balthazar et al., 2014b; Rodrigues et al., 2014), nonideal systems with chaotic behavior (Tusset et al., 2012b, 2012c) and control in nonlinear structural dynamical systems in the presence of noise (Sajeeb et al., 2007).

Motivated by the necessity to improve passenger comfort levels, our research aims to study and control the dynamic horizontal behavior of a three-degree-offreedom model of a vertical transportation system excited by guide rail deformations. The translational equivalent stiffness of the spring referring the tilting motion of the cabin is modeled as a Duffing-type spring. With the use of the Duffing-type spring it is possible to analyze the small horizontal displacement of the elevator and the strain force of the vertical cable, similar to a hardening spring. As a strategy to control the horizontal vibrations, the SDRE method is used. Next, we perform extensive numerical simulation studies of the nonlinear behavior of the adopted mathematical model.

This paper is organized as follows. The mathematical model of the vertical transportation system is shown in Section 2. Results and discussion of the uncontrolled model are shown in Section 3. The control system design using the SDRE strategy with and without the presence of parametric errors and noise measurements is shown in Section 4. Finally, conclusions are drawn in Section 5.

\section{Mathematical modeling}

Figure 1 shows a schematic diagram of the cabin elevator. The roller guides support the platform with springs to avoid the transmission of external excitation caused by misalignment and deformation of guide rails. These roller guides and springs are components of the suspension system. Platform rubber elements are attached between the cabin and the frame to isolate vibrations.

Figure 2 shows an equivalent physical model to represent the horizontal motions of an elevator system.

In a previous work, Lopez et al. (2010) proposed the physical parameters for a similar model to the one presented in this paper for the horizontal motion of the elevator (Figure 2) obtained from the model represented in Figure 1. Funai et al. (2004) showed experimental data obtained on the horizontal acceleration for

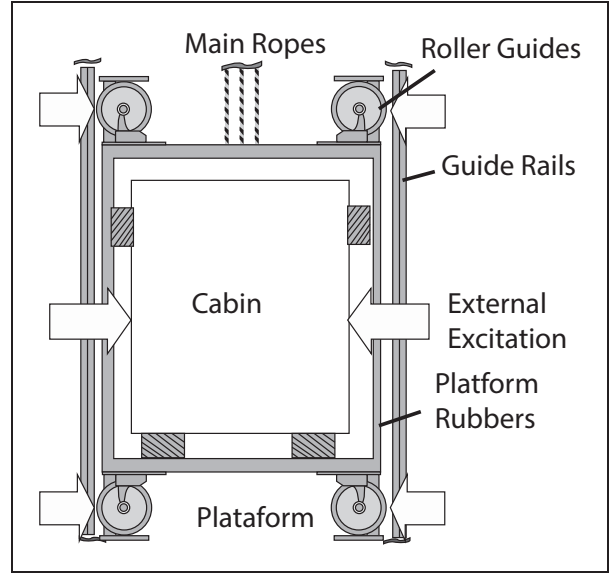

Figure I. Schematic diagram of the cabin structure.

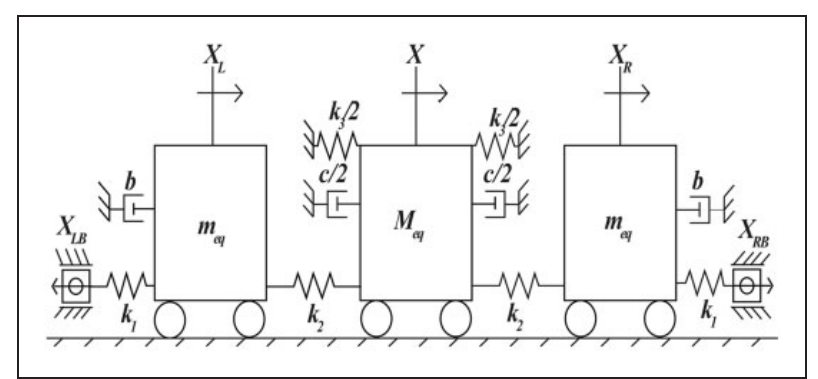

Figure 2. Equivalent model for the horizontal motion of the elevator.

the model shown in Figure 1, allowing, thus, one to examine the horizontal acceleration of the elevator, obtained by numerical simulation considering the parameters proposed by Lopez et al. (2010), and the horizontal acceleration of the elevator obtained from experimental data of Funai et al. (2004). In Figure 2, $M_{\text {eq }}$ is the mass of the cabin $(\mathrm{kg}), m_{\text {eq }}$ is the mass of the suspension system $(\mathrm{kg}), b$ is the damping coefficient of the suspension $(\mathrm{N} \mathrm{s} / \mathrm{m}), c$ is the damping coefficient of the cabin $(\mathrm{N} \mathrm{s} / \mathrm{m}), k_{1}$ is the stiffness coefficient of the guide rollers $(\mathrm{N} / \mathrm{m}), k_{2}$ is the stiffness coefficient of the suspension $(\mathrm{N} / \mathrm{m}), k_{3}$ is the stiffness coefficient of the elevator ropes referring to the tilting motion of the cabin $(\mathrm{N} / \mathrm{m}), X$ is the displacement of the cabin $(\mathrm{m})$, $X_{\mathrm{L}}$ is the displacement of the left-hand suspension system $(\mathrm{m})$ and $X_{\mathrm{R}}$ is the displacement of the righthand suspension system (m). $X_{\mathrm{LB}}$ and $X_{\mathrm{RB}}$ are external excitations caused by guide rail deformations, defined in equation (1)

$$
X_{\mathrm{LB}}=X_{\mathrm{RB}}=a_{0} \sin \left(\omega_{\mathrm{e}} t\right)
$$

$a_{0}$ is the amplitude of external excitation $(\mathrm{m})$ and $\omega_{\mathrm{e}}$ is the external excitation frequency $(\mathrm{rad} / \mathrm{s})$. In order to analyze the nonlinearity of the stiffness coefficient of 
the elevator ropes, the parameter $\left(k_{3}\right)$ will represent the Duffing spring stiffness coefficient, being obtained by the sum of the linear and nonlinear terms $k_{3 \mathrm{~L}}(\mathrm{~N} / \mathrm{m})$ and $k_{3 \mathrm{NL}}\left(\mathrm{N} / \mathrm{m}^{3}\right.$ ), respectively, as follows (Kovacic and Brennan, 2011; Carrella et al., 2012; BeltránCarbajal and Silva-Navarro, 2014; Khan et al., 2014; Tu et al., 2015)

$$
k_{3}=k_{3 \mathrm{~L}}+k_{3 \mathrm{NL}} X^{2}
$$

Lopez et al. (2010) used equation (2) without the $k_{3 \mathrm{NL}}$ term, that is, parameter $k_{3 \mathrm{~L}}=6662.4(\mathrm{~N} / \mathrm{m})$, whereas $k_{3 \mathrm{NL}}=0\left(\mathrm{~N} / \mathrm{m}^{3}\right)$. However, when in the upright position, there is then no traction on the cable in the horizontal direction, and the horizontal restoring force only appears when there is a small lateral displacement of the elevator and the tension force of the vertical cable has a small horizontal component, similar to a hardening spring. Hence, the restoring force of the cable does not have a constant term, that is, $k_{3 \mathrm{~L}}=0(\mathrm{~N} / \mathrm{m})$.

In this regard, the potential energy of the spring $\left(E_{\mathrm{pel}}\right)$ and the restitution force $\left(F_{\mathrm{el}}\right)$ associated with the nonlinear Duffing spring are as shown in equations (3) and (4), respectively (Kovacic and Brennan, 2011; Carrella et al., 2012; Beltrán-Carbajal and Silva-Navarro, 2014; Khan et al., 2014; Tu et al., 2015)

$$
\begin{gathered}
E_{\mathrm{Pel}}=\frac{1}{2} k_{3 \mathrm{~L}} X^{2}+\frac{1}{4} k_{3 \mathrm{NL}} X^{4} \\
F_{\mathrm{el}}=k_{3 \mathrm{~L}} X+k_{3 \mathrm{NL}} X^{3}
\end{gathered}
$$

The Kinect $(T)$ and Potential $(V)$ energies of the system, shown in Figure 2, are represented in equations (5) and (6), respectively

$$
\begin{gathered}
T=\frac{m_{\mathrm{eq}}}{2} \dot{X}_{\mathrm{L}}^{2}+\frac{M_{\mathrm{eq}}}{2} \dot{X}^{2}+\frac{m_{\mathrm{eq}}}{2} \dot{X}_{\mathrm{R}}^{2} \\
V=\frac{k_{1}}{2}\left(X_{\mathrm{L}}-X_{\mathrm{LB}}\right)^{2}+\frac{k_{1}}{2}\left(X_{\mathrm{RB}}-X_{\mathrm{R}}\right)^{2}+\frac{k_{2}}{2}\left(X-X_{\mathrm{L}}\right)^{2} \\
+\frac{k_{2}}{2}\left(X_{\mathrm{R}}-X\right)^{2}+\frac{1}{2} k_{3 \mathrm{~L}} X^{2}+\frac{1}{4} k_{3 \mathrm{NL}} X^{4}
\end{gathered}
$$

The sum of conservative and nonconservative generalized forces $\left(Q_{k}\right)$ of the system can be written as follows

$$
Q_{k}=b \dot{X}_{\mathrm{L}}+b \dot{X}_{\mathrm{R}}+c \dot{X}
$$

Considering the Lagrangian (Meirovitch, 1970)

$$
L=T-V
$$

The equations of motion that represent the vertical transportation can be obtained from (Meirovitch, 1970)

$$
\frac{d}{d t}\left(\frac{\partial L}{\partial \dot{q}_{i}}\right)-\frac{\partial L}{q_{i}}=Q_{k} i
$$

where $i=1,2,3, \quad q_{1}=X_{\mathrm{L}}, \quad q_{2}=X, \quad q_{3}=X_{\mathrm{R}}$, $Q_{\mathrm{k}} 1=b \dot{X}_{\mathrm{L}}, Q_{\mathrm{k}} 2=b \dot{X}_{\mathrm{R}}$ and $Q_{\mathrm{k}} 3=c \dot{X}$.

Using the Lagrange formulation (equations (8) and (9)) and considering equations (5)-(7) and $k_{3 \mathrm{~L}}=0$, the second-order differential equations of the system can be represented by equation (10)

$$
\begin{aligned}
m_{\mathrm{eq}} \ddot{X}_{\mathrm{L}}+b \dot{X}_{\mathrm{L}}+\left(k_{1}+k_{2}\right) X_{\mathrm{L}}-k_{2} X & =k_{1} X_{\mathrm{LB}} \\
M_{\mathrm{eq}} \ddot{X}+c \dot{X}+2 k_{2} X+k_{3 \mathrm{NL}} X^{3}-k_{2} X_{\mathrm{L}}-k_{2} X_{\mathrm{R}} & =0 \\
m_{\mathrm{eq}} \ddot{X}_{\mathrm{R}}+b \dot{X}_{\mathrm{R}}+\left(k_{1}+k_{2}\right) X_{\mathrm{R}}-k_{2} X & =k_{1} X_{\mathrm{RB}}
\end{aligned}
$$

In order to obtain dimensionless equations of motion, we introduce the following dimensionless variables: $y_{1}=\frac{X_{\mathrm{L}}}{X_{\text {est }}}, y_{3}=\frac{X}{X_{\text {est }}}, y_{5}=\frac{X_{\mathrm{R}}}{X_{\text {est }}}$ and $\tau=\omega_{0} t$, where $X_{\text {est }}(\mathrm{m})$ is a constant used in dimensionless equations (Nayfeh, 1973). Then, introducing equation (1) into equation (10) and combining with the dimensionless variables, the dimensionless equations of motion can be written in state-space representation as follows

$$
\begin{aligned}
& \dot{x}_{1}=x_{2} \\
& \dot{x}_{2}=-\zeta x_{2}-\delta x_{1}+\alpha x_{3}+\gamma \sin (\Omega \tau) \\
& \dot{x}_{3}=x_{4} \\
& \dot{x}_{4}=-\sigma x_{4}-2 \eta x_{3}-\beta x_{3}^{3}+\eta x_{1}+\eta x_{5} \\
& \dot{x}_{5}=x_{6} \\
& \dot{x}_{6}=-\zeta x_{6}-\delta x_{5}+\alpha x_{3}+\gamma \sin (\Omega \tau)
\end{aligned}
$$

where $x_{1}=y_{1}, \quad x_{2}=\dot{y}_{1}, \quad x_{3}=y_{3}, \quad x_{4}=\dot{y}_{3}, \quad x_{5}=y_{5}$, $x_{6}=\dot{y}_{5}, \quad \zeta=\frac{b}{m_{\mathrm{eq}} \omega_{0}}, \quad \delta=\frac{k_{1}+k_{2}}{m_{\mathrm{eq}} \omega_{0}^{2}}, \quad \alpha=\frac{k_{2}}{m_{\mathrm{eq}} \omega_{0}^{2}}, \quad \gamma=\frac{k_{1} a_{0}}{m_{\mathrm{eq}} \omega_{0}^{2} x_{\mathrm{est}}}$, $\eta=\frac{k_{2}}{M_{\mathrm{eq}} \omega_{0}^{2}}, \beta=\frac{k_{3 \mathrm{NL}} X_{\mathrm{est}}^{2}}{M_{\mathrm{eq}} \omega_{0}^{2}}, \sigma=\frac{c}{M_{\mathrm{eq}} \omega_{0}}, \Omega=\frac{\omega_{\mathrm{e}}}{\omega_{0}}$ and $\omega_{0}=\sqrt{\frac{k_{1}}{m_{\mathrm{eq}}}}$.

\section{Dynamic behavior of the uncontrolled system}

In this section, we present numerical simulations of the dynamic behavior of the uncontrolled system represented in equation (11). In order to simulate the influence of the traction cable on the dynamics system of the model, various features of the dynamical behavior of a cabin elevator for different values of the parameter $\beta$ are presented. For this purpose, we performed computational analyses based on the bifurcation diagram, Lyapunov exponents and Kaplan-Yorke dimensions. Table 1 shows the parameter values used in the numerical simulations, considering the physical parameters 
Table I. Parameters used for numerical simulations.

\begin{tabular}{ll}
\hline Parameter & \\
\hline$\zeta$ & 0.3195 \\
$\delta$ & 1.0761 \\
$\alpha$ & 0.0761 \\
$\gamma$ & 0.005 \\
$\eta$ & 0.0012 \\
$\sigma$ & 0.0154 \\
$\Omega$ & 0.2628 \\
\hline
\end{tabular}

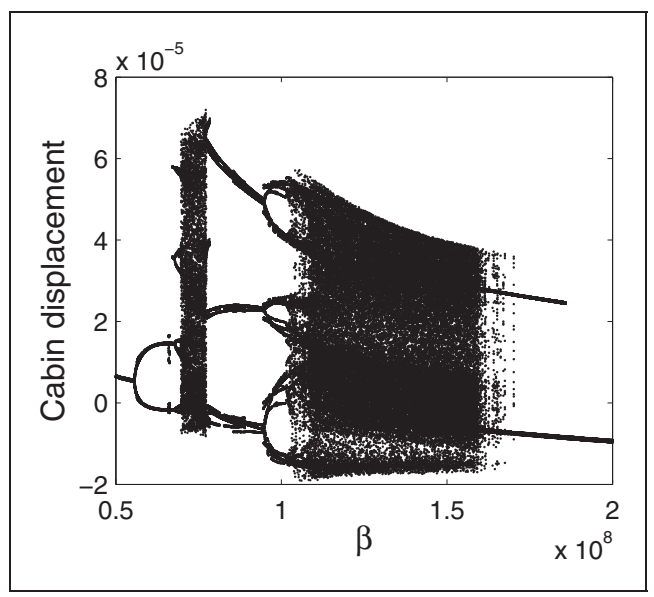

Figure 3. Bifurcation diagram for the $\beta$ parameter from $0.5 \times 10^{8}$ to $2.1 \times 10^{8}$.

$m_{\mathrm{eq}}=17.5(\mathrm{~kg}), M_{\mathrm{eq}}=1120(\mathrm{~kg}), k_{1}=250,000(\mathrm{~N} / \mathrm{m})$, $k_{2}=19,027(\mathrm{~N} / \mathrm{m}), \quad b=668.21(\mathrm{Ns} / \mathrm{m}), \quad c=2058.2$ $(\mathrm{Ns} / \mathrm{m}), a_{0}=0.005(\mathrm{~m}), \omega_{\mathrm{e}}=0.2628445(\mathrm{rad} / \mathrm{s})$ and $X_{\text {est }}=1(\mathrm{~m})$. The physical parameters used in the numerical simulations are similar to those obtained by Lopez et al. (2010).

Figure 3 shows the bifurcation diagram obtained by varying the parameter $\beta=\frac{k_{3 \mathrm{NL}} X_{\text {st }}^{2}}{M_{\mathrm{eq}} \omega_{0}^{2}}$ that is related to the nonlinear term of the Duffing spring $\left(k_{3 \mathrm{NL}}\right)$. For this case, we may observe a period-doubling route to chaos and strange attractors as we increase parameter $\beta$.

In Figure 3, it can be observed that the behavior of the cabin varies the beta parameter, considering the proportional change to the nonlinear term of the Duffing spring $\left(k_{3 \mathrm{NL}}=\left[1 \times 10^{14}, 3.35 \times 10^{14}\right]\left(\mathrm{N} / \mathrm{m}^{3}\right)\right)$. The values considered for $\left(k_{3 \mathrm{NL}}\right)$ generate potential energy and strength similar to obtained by Lopez et al. (2010), considering only the constant term $\left(k_{3 \mathrm{~L}}=6662.4(\mathrm{~N} / \mathrm{m})\right)$. The variation $\left(k_{3 \mathrm{NL}}\right)$ can be related to different diameters of the elevator ropes.

Our state space is composed of six states (equation (11)); therefore, the system has six Lyapunov exponents, one of which is always zero, in the direction

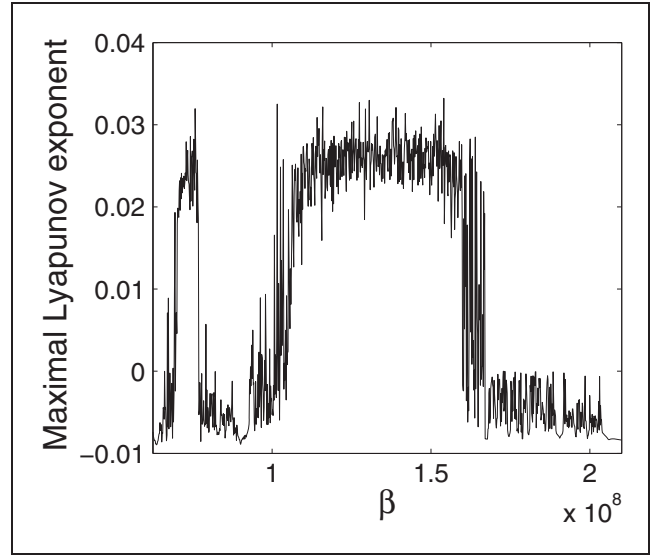

Figure 4. Maximum Lyapunov exponent $\left(\beta=\left[6.25 \times 10^{7}, 2.1 \times 10^{8}\right]\right)$.

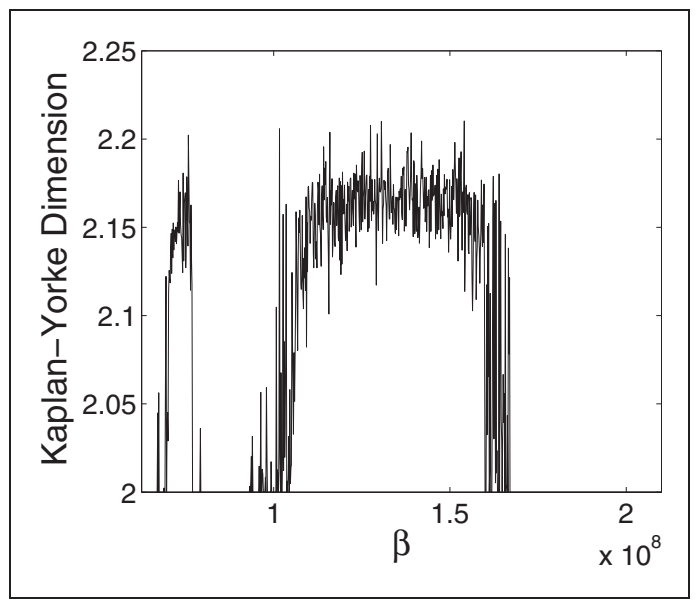

Figure 5. Kaplan-Yorke dimension $\left(\beta=\left[6.25 \times 10^{7}, 2.1 \times 10^{8}\right]\right)$.

tangent to the flow. Regarding the other five exponents, the maximum Lyapunov exponent is less than zero for a stable periodic orbit, whereas for a chaotic orbit the maximum Lyapunov exponent is greater than zero.

Figure 4 shows the maximal Lyapunov exponent as a function of $\beta$ for the bifurcation diagram given by Figure 3, computed using the Wolf algorithm (Wolf et al., 1985). As can be seen, there is a correspondence between the bifurcation plots and the maximal Lyapunov exponent curve.

Figure 5 shows the result of the Kaplan-Yorke dimension of the two possible attractors of the cabin dynamics.

The Kaplan-Yorke dimension (Kaplan and Yorke, 1979) is calculated as follows

$$
D_{\mathrm{KY}}=m+\frac{\sum_{n=l}^{m} \lambda_{l}}{\lambda_{m+1}}
$$




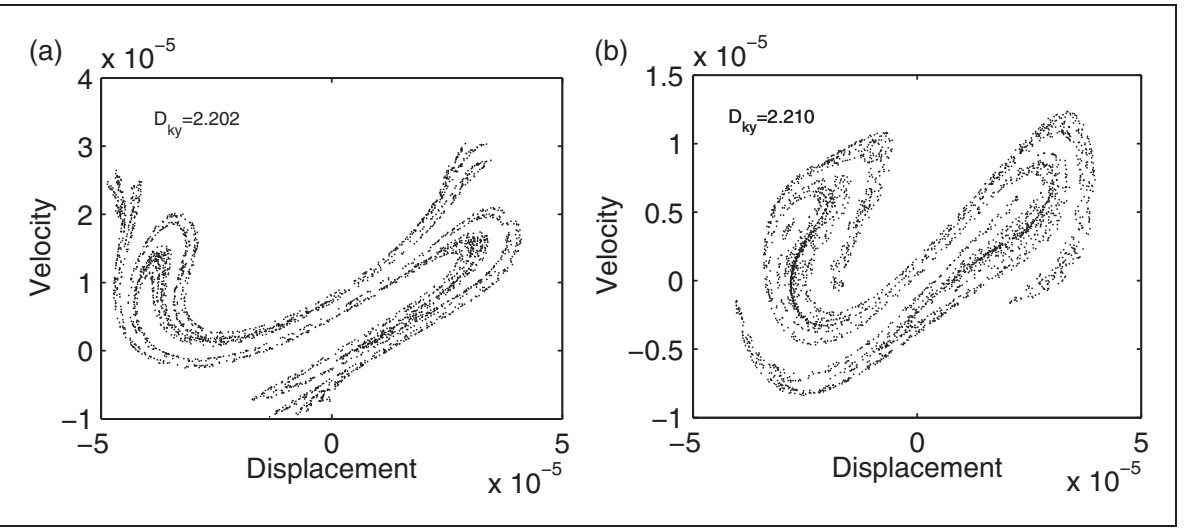

Figure 6. Poincaré map for (a) $\beta=7.578 \times 10^{7}$ and (b) $\beta=1.54 \times 10^{8}$.

where $m \leq n$ is the largest index such that $\sum_{n=l}^{m} \lambda_{l} \geq 0$.

The dimensions of the chaotic attractor are always between 2 and 3, that is, the space attractor is topologically complex: more than a limit cycle and less than a threedimensional object (Sharma et al., 2012). In order to compare the geometrical complexity of the phase space attractors, the Kaplan-Yorke dimension was calculated. Figure 6 shows the Poincare map for the two different chaotic attractors of the system, and it is possible to note the complexity of these phase space attractors.

In Figure 6(a) it is possible to observe the first type of attractor obtained for this system. All the attractors in the interval $\beta \in\left[6.69 \times 10^{7}, 7.69 \times 10^{7}\right]$ have the topology shown in Figure 6(a). On the other hand, the second type of attractor shows the highest KaplanYorke dimension obtained for the system, Figure 6(b), when $\beta$ is in the interval $\left[9.3 \times 10^{7}, 1.88 \times 10^{8}\right]$. The results of Kaplan-Yorke highlight the geometrical complexity/fractalness of the phase space attractor when increase of the parameter $\beta$ occurs.

\section{Nonlinear control design using the State-dependent Ricatti Equation technique}

The control objective is to reduce the magnitude of the cabin's acceleration and displacement in order to contribute to the preservation of the elevator's component integrity and passenger comfort level. Consider now the introduction of an active controller damping parallel to the roller guide spring $k_{2}$. In this way, the system given by equation (6), with the introduction of active control, can be represented as

$$
\begin{aligned}
& \dot{x}_{1}=x_{2} \\
& \dot{x}_{2}=-\zeta x_{2}-\delta x_{1}+\alpha x_{3}+\gamma \sin (\Omega \tau)+\kappa U \\
& \dot{x}_{3}=x_{4}
\end{aligned}
$$

$$
\begin{aligned}
& \dot{x}_{4}=-\sigma x_{4}-2 \eta x_{3}-\beta x_{3}^{3}+\eta x_{1}+\eta x_{5}-\chi U \\
& \dot{x}_{5}=x_{6} \\
& \dot{x}_{6}=-\zeta x_{6}-\delta x_{5}+\alpha x_{3}+\gamma \sin (\Omega \tau)
\end{aligned}
$$

where $\kappa=\frac{1}{m_{\mathrm{eq}} \omega_{0}^{2}}$ and $\chi=\frac{1}{M_{\mathrm{eq}} \omega_{0}^{2}}$.

It is possible to rewrite equation (13) in the following form

$$
\dot{\mathbf{x}}=\mathbf{A x}+\mathbf{B U}+\mathbf{G}
$$

where $\mathbf{x} \in \Re^{6}$ is a state vector, $\mathbf{A} \in \mathfrak{R}^{6 \times 6}$ is the matrix of state variables, $\mathbf{B} \in \Re^{6}$ is a matrix of controller gains, $\mathbf{U} \in \Re$ represents the control vector and $\mathbf{G} \in \Re^{6}$ is the vector that represents external perturbations, which do not depend on the states $(\mathbf{x})$. Thus

$$
\begin{gathered}
\mathbf{A}=\left[\begin{array}{cccccc}
0 & 1 & 0 & 0 & 0 & 0 \\
-\delta & -\zeta & \alpha & 0 & 0 & 0 \\
0 & 0 & 0 & 1 & 0 & 0 \\
\eta & 0 & -2 \eta-\beta x_{3}^{2} & -\sigma & \eta & 0 \\
0 & 0 & 0 & 0 & 0 & 1 \\
0 & 0 & \alpha & 0 & -\delta & -\zeta
\end{array}\right] \\
\mathbf{B}=\left[\begin{array}{c}
0 \\
\kappa \\
0 \\
-\chi \\
0 \\
0
\end{array}\right] \\
\mathbf{G}=\left[\begin{array}{c}
0 \\
\gamma \sin (\Omega \tau) \\
0 \\
0 \\
0 \\
\gamma \sin (\Omega \tau)
\end{array}\right]
\end{gathered}
$$


Tusset et al. (2013) gave the following state feedback control law

$$
\mathbf{U}=\mathbf{R}^{-1} \mathbf{B}^{\mathbf{T}} \mathbf{P} \mathbf{x}
$$

where $\mathbf{P}$ is the solution to the SDRE, given by Tusset et al. (2012a)

$$
\mathbf{A}^{\mathrm{T}} \mathbf{P}+\mathbf{P A}-\mathbf{P B R}^{-1} \mathbf{B}^{\mathrm{T}} \mathbf{P}+\mathbf{Q}=\mathbf{0}
$$

The cost function for the regulator problem in equation (18) is given by Tusset et al. (2012c)

$$
J=\frac{1}{2} \int_{t_{0}}^{\infty}\left[\mathbf{x}^{\mathrm{T}} \mathbf{Q} \mathbf{x}+\mathbf{U}^{\mathrm{T}} \mathbf{R} \mathbf{U}\right] \mathrm{d} \tau
$$

where $\mathbf{Q}$ and $\mathbf{R}$ are symmetric positive definite matrices. Ogata (2009) describes that the matrices $\mathbf{Q}$ and $\mathbf{R}$ should be positive definite, and this is a necessary and sufficient condition for $\mathbf{P}$ in (equation (19)) being symmetric positive definite.

The choice of $\mathbf{Q}$ and $\mathbf{R}$ is very crucial to the stabilization and performance of the system. For example, as the values of $\mathbf{Q}$ increase, the greater will be the control input and smaller the time taken to reduce perturbations of the systems, since matrix $\mathbf{P}$ used for the control signal equation (18) depends on the value of $\mathbf{Q}$, which can be seen in equation (19). Increasing the values of $\mathbf{R}$ causes a decrease in the values of feedback gain, because the gain control equation (18) depends on the inverse of $\mathbf{R}$, as can be observed in the control equation (18) (Singh et al., 2008).

\section{I. Application of SDRE control}

The control signal $\mathbf{U}$ is determined using $\kappa=4 \times 10^{-6}$, $\chi=6.25 \times 10^{-8}$, matrices $\mathbf{A}$ and $\mathbf{B}$, and defining the symmetric positive definite matrices $\mathbf{Q}$ and $\mathbf{R}$

$$
\mathbf{Q}(\mathbf{x})=10^{7}\left[\begin{array}{cccccc}
1 & 0 & 0 & 0 & 0 & 0 \\
0 & 1 & 0 & 0 & 0 & 0 \\
0 & 0 & 10000 & 100 & 0 & 0 \\
0 & 0 & 100 & 10000 & 0 & 0 \\
0 & 0 & 0 & 0 & 1 & 0 \\
0 & 0 & 0 & 0 & 0 & 1
\end{array}\right]
$$

For simplicity we will restrict ourselves to using only constant values for matrices $\mathbf{Q}$ and $\mathbf{R}$, similar to the strategy used by Singh et al. (2008) and Tusset et al. (2015). The matrix $\mathbf{Q}$ was selected to reduce perturbations on states $x_{3}$ and $x_{4}$ (cabin), penalizing the states $x_{1}, x_{2}, x_{5}$ and $x_{6}$ (guide rollers), and $\mathbf{R}$ was used to reduce the time stabilization. The performance of the controller obtained by the SDRE technique can be modified through the tuning of the $\mathbf{Q}$ and $\mathbf{R}$.

Another important factor to consider is that matrix A cannot violate the controllability of the system. The system shown in equation (14) is controllable if the rank of matrix $\mathbf{M}$ is 6

$$
\mathbf{M}=\left[\begin{array}{llll}
\mathbf{B}_{6 \times 1} & \mathbf{A}_{6 \times 6} \mathbf{B}_{6 \times 1} & \cdots & \mathbf{A}_{6 \times 6}^{5} \mathbf{B}_{6 \times 1}
\end{array}\right]
$$

The SDRE technique to obtain a solution for the dynamic control problem has the following procedure (Tusset et al., 2015).

1. Define the state-space model with the state-dependent coefficient as in equation (15).

2. Define $\mathbf{x}(\mathbf{0})=\mathbf{x}_{\mathbf{0}}$, so that the rank of $\mathbf{M}$ is (rank $\left.=6\right)$ and choose the coefficients of weight matrices $\mathbf{Q}$ and $\mathbf{R}$.

3. Solve the Riccati Equation (19) for state $\mathbf{x}$.

4. Calculate the input signal from equation (18).

5. Integrate equation (14) and update the state of the system $\mathbf{x}$ with this result.

6. Calculate the rank of equation (23); if rank $=6$ go to Step 3. However, if rank $<6$, matrix $\mathbf{A}$ is not controllable; therefore, one should use the last matrix controllable A that has been obtained, and thus go to Step 3.

Figures 7 and 8 show the comparison of the behavior of the system without control and with the SDRE control strategy, when $\beta=7.578 \times 10^{7}$ and $\beta=1.54 \times 10^{8}$, respectively.

It is possible to observe that the proposed SDRE control reduced the system's oscillation amplitude (Figures 7(a) and 8(a)). In addition, the SDRE control has driven the system dynamics to a periodic orbit (see Figures 7(b) and 8(b)). We can measure the performance of control using the root mean square (RMS) of the controlled system. The RMS of the time history of the acceleration shown in Figure $7(\mathrm{c})$ is $\left(\ddot{x}_{3_{R M S}} \approx 1.001 \times 10^{-5}\left[\mathrm{~ms}^{-2}\right]\right)$ and the time history of the jerk shown in Figure 7(d) is $\left(\dddot{x}_{3_{R M S}} \approx 1.0403 \times 10^{-5}\left[\mathrm{~ms}^{-3}\right]\right)$ for the system without control and $\left(\ddot{x}_{3_{R M S}} \approx 3.8288 \times 10^{-6}\left[\mathrm{~ms}^{-2}\right]\right)$ and $\left(\dddot{x}_{3_{R M S}} \approx 1.9107 \times 10^{-6}\left[\mathrm{~ms}^{-3}\right]\right)$ for the system with control. The RMS of the response shown in Figures $8(\mathrm{c})$ and $8(\mathrm{~d})$ are $\left(\ddot{x}_{3_{R M S}} \approx 1.029 \times 10^{-5}\left[\mathrm{~ms}^{-2}\right]\right.$ and $\left.\dddot{x}_{3_{R M S}} \approx 1.1252 \times 10^{-5}\left[\mathrm{~ms}^{-3}\right]\right)$ for the system without control and $\left(\ddot{x}_{3_{R M S}} \approx 4.629 \times 10^{-6}\left[\mathrm{~ms}^{-2}\right]\right.$ and $\left.\dddot{x}_{3_{R M S}} \approx 6.0432 \times 10^{-7}\left[\mathrm{~ms}^{-3}\right]\right)$ for the system with control. The RMS of the acceleration and of the jerk are reduced in about $62.09 \%$ and $95.21 \%$ for $\left(\beta=7.578 \times 10^{7}\right), \quad 55.01 \%$ and $94.63 \%$ for 


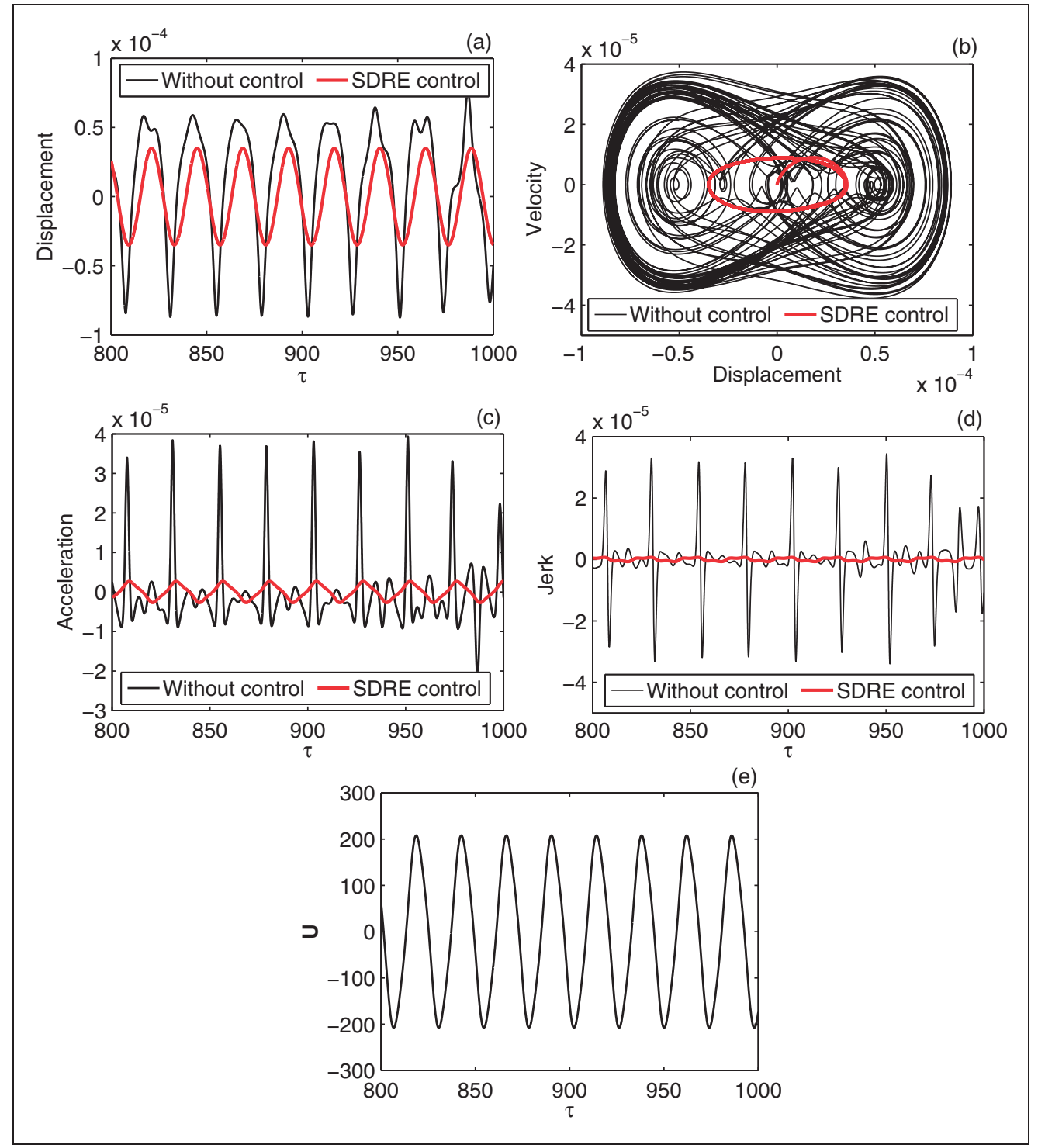

Figure 7. Comparison between uncontrolled (black) and controlled system using the State-dependent Ricatti Equation (SDRE) technique (red) cabin response for $\beta=7.578 \times 10^{7}$ : (a) history of the displacement; (b) phase plane; (c) history of acceleration; (d) history of jerk; (e) signal used in SDRE control (damping [Ns/m]).

$\left(\beta=1.54 \times 10^{8}\right)$ in Figures $7(\mathrm{c})$ and (d) and Figures 8(c) and $8(d)$, respectively. The values of the RMS of the acceleration obtained here are very close to acceleration range observed by Funai et al. (2004) for an experimental model considering the schematic diagram of the cabin structure model (Figure 1).

The variations of the control signal used in the SDRE technique, for both values of $\beta$, are presented in Figures 7(d) and 8(d), respectively. Because the control signal has low values, it can be considered as a magnetorheological, pneumatic, hydraulic or even an electromagnetic actuator. The application of a magnetorheological damper can control the damping force by the applied electric current, as applied by Tusset et al. (2012b, 2012c). An electromagnetic actuator can be used as in the strategy of the Oh et al. (2006), which used active control based on the repulsive forces of electromagnets to reduce lateral disturbances, or the strategy of the Utsunomiya et al. (2004, 2006), which used adequate lateral forces through electromagnetic actuators to reduce lateral disturbances of the elevator.

\subsection{Controlled system in the presence of parametric errors and noise measurements}

Control designs based on the parameters of a certain mathematical model will often not represent the real dynamics (Tusset et al., 2015). Consequently, 


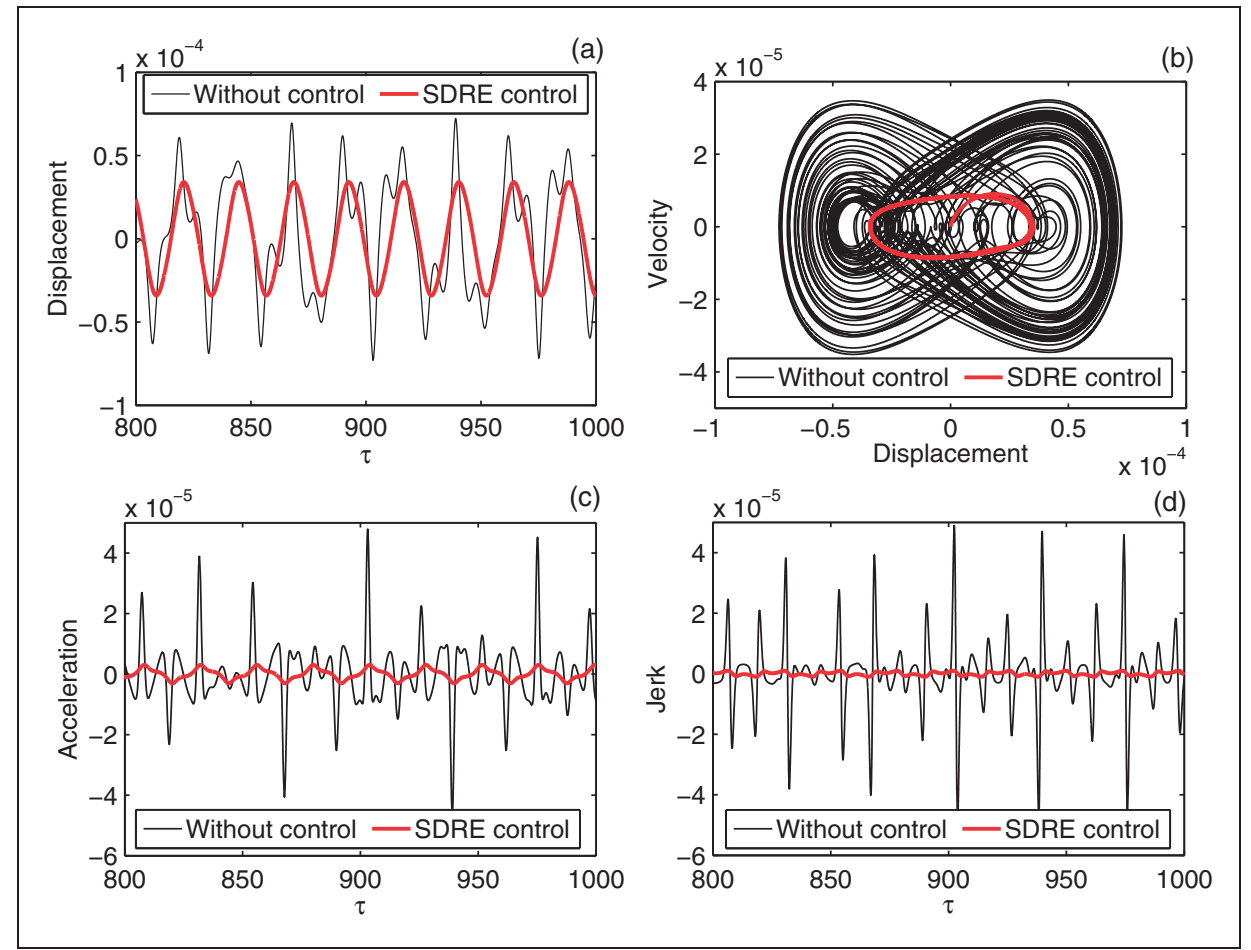

Figure 8. Comparison between uncontrolled (black) and controlled system using the State-dependent Ricatti Equation (SDRE) technique (red) cabin response for $\beta=1.54 \times 10^{8}$ : (a) history of the displacement; (b) phase plane; (c) history of acceleration; (d) history of jerk; (e) signal used in SDRE control (damping [Ns/m]).

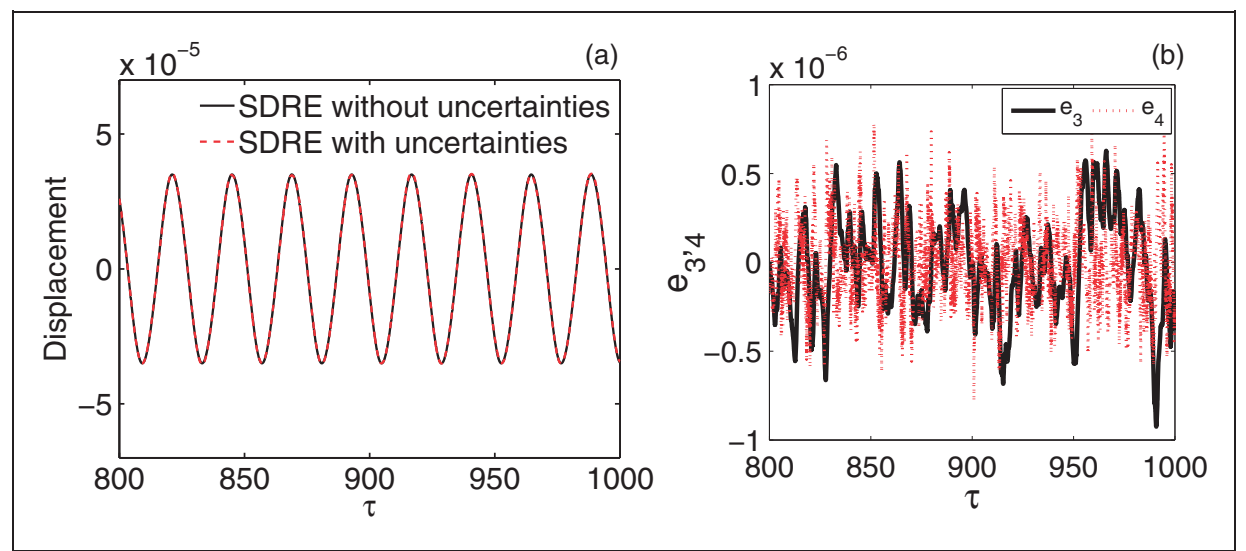

Figure 9. Parameter uncertainties in A for $\beta=7.578 \times 10^{7}$ : (a) State-dependent Ricatti equation (SDRE) control displacement with and without uncertainties; (b) deviation in displacement $\left(e_{3}=x_{3}-\hat{x}_{3}\right)$ and deviation in velocity $\left(e_{4}=x_{4}-\hat{x}_{4}\right)$ error for uncertainty in parameters.

the control design will not operate as intended when applied in a real process, because the parameters used in the control may contain parametric uncertainties. The parametric uncertainties are associated with discrepancies between system actual physical values and the numerical parameters used in the analysis. To solve this problem, researches have focused on incorporating the uncertainties associated with real systems into numerical simulations for reliable predictions (Triguero et al., 2013). To consider uncertain parameter effects on the control performance, parameters $\delta, \zeta, \alpha, \eta, \beta, \sigma, \gamma, \kappa$ and $\chi$ used in the matrix of state variables $\mathbf{A}$, the matrix 


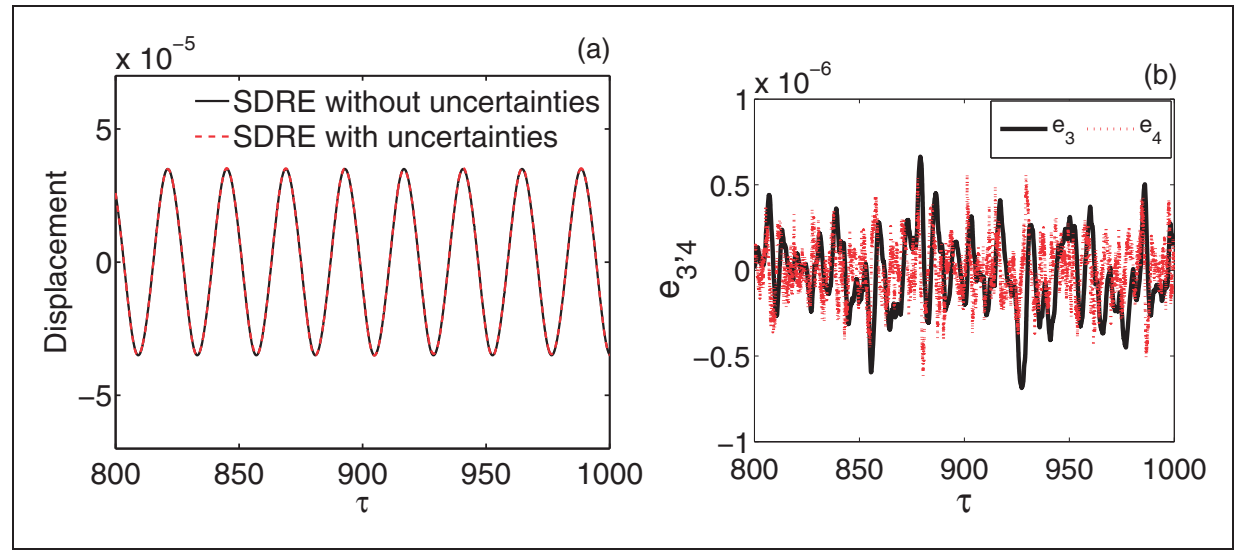

Figure 10. Parameter uncertainties in $\mathbf{A}$ and $\mathbf{G}$ for $\beta=7.578 \times 10^{7}$ : (a) State-dependent Ricatti Equation (SDRE) control displacement with and without uncertainties; (b) deviation in displacement $\left(e_{3}=x_{3}-\hat{x}_{3}\right)$ and deviation in velocity $\left(e_{4}=x_{4}-\hat{x}_{4}\right)$ error for uncertainty in parameters.

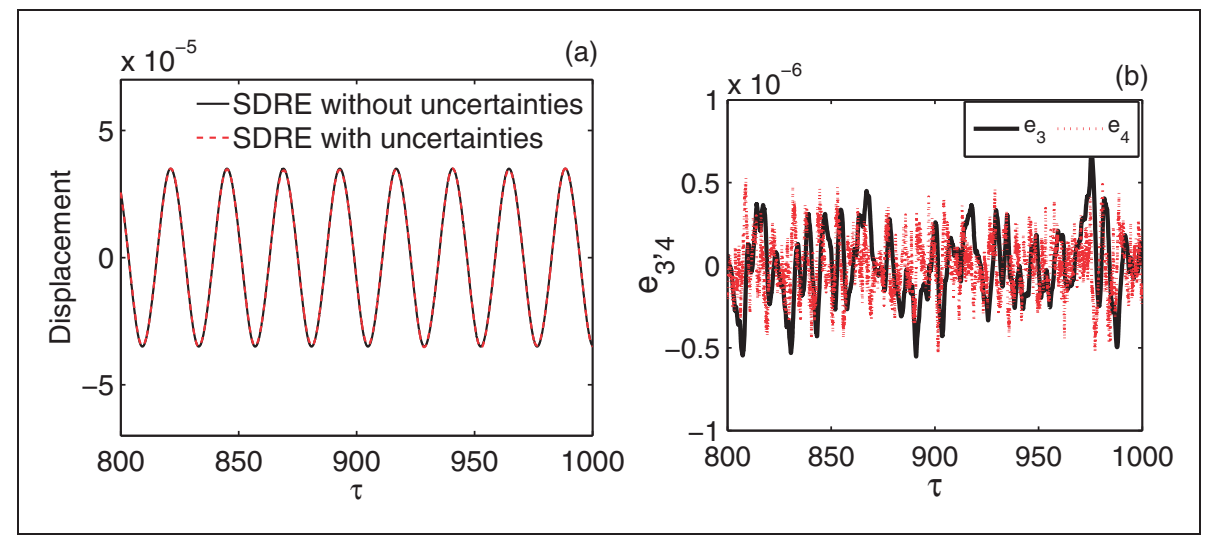

Figure II. Parameter uncertainties in A, G and $\mathbf{B}$ for $\beta=7.578 \times 10^{7}$ : (a) State-dependent Ricatti Equation (SDRE) control displacement with and without uncertainties; (b) deviation in displacement $\left(e_{3}=x_{3}-\hat{x}_{3}\right)$ and deviation in velocity $\left(e_{4}=x_{4}-\hat{x}_{4}\right)$ error for uncertainty in parameters.

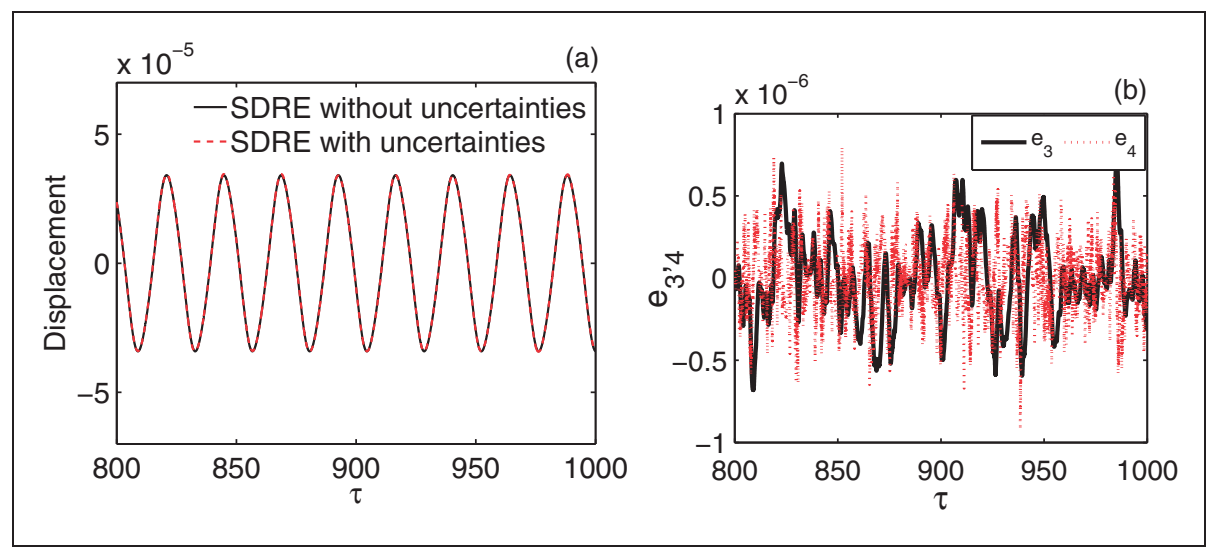

Figure 12. Parameter uncertainties in A for $\beta=1.54 \times 10^{8}$ : (a) State-dependent Ricatti Equation (SDRE) control displacement with and without uncertainties; (b) deviation in displacement $\left(e_{3}=x_{3}-\hat{x}_{3}\right)$ and deviation in velocity $\left(e_{4}=x_{4}-\hat{x}_{4}\right)$ error for uncertainty in parameters. 


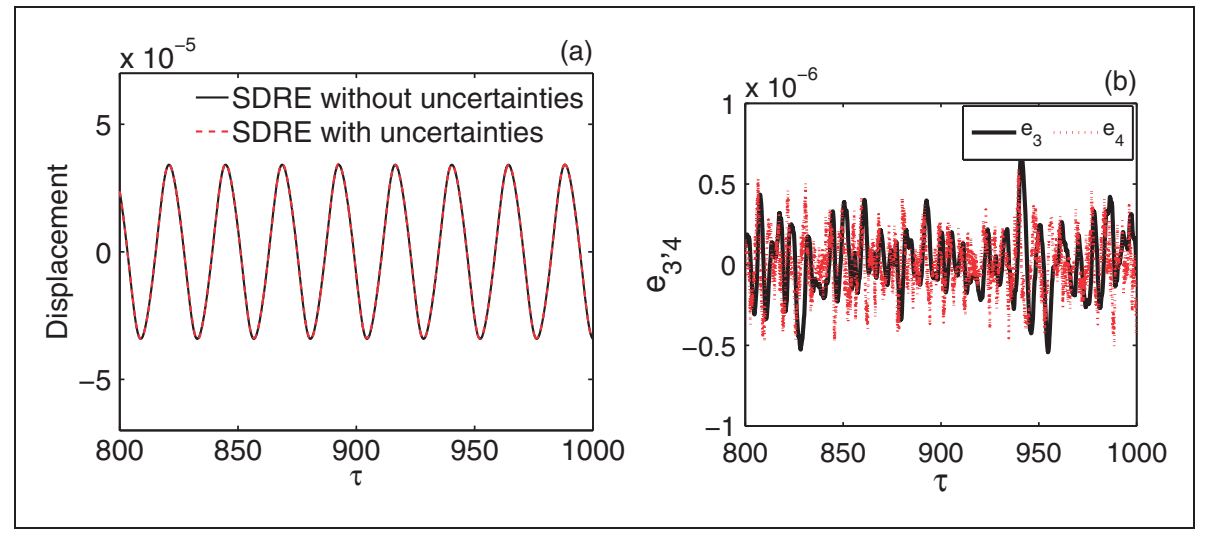

Figure 13. Parameter uncertainties in $\mathbf{A}$ and $\mathbf{G}$ for $\beta=1.54 \times 10^{8}$ : (a) State-dependent Ricatti Equation (SDRE) control displacement with and without uncertainties; (b) deviation in displacement $\left(e_{3}=x_{3}-\hat{x}_{3}\right)$ and deviation in velocity $\left(e_{4}=x_{4}-\hat{x}_{4}\right)$ error for uncertainty in parameters.

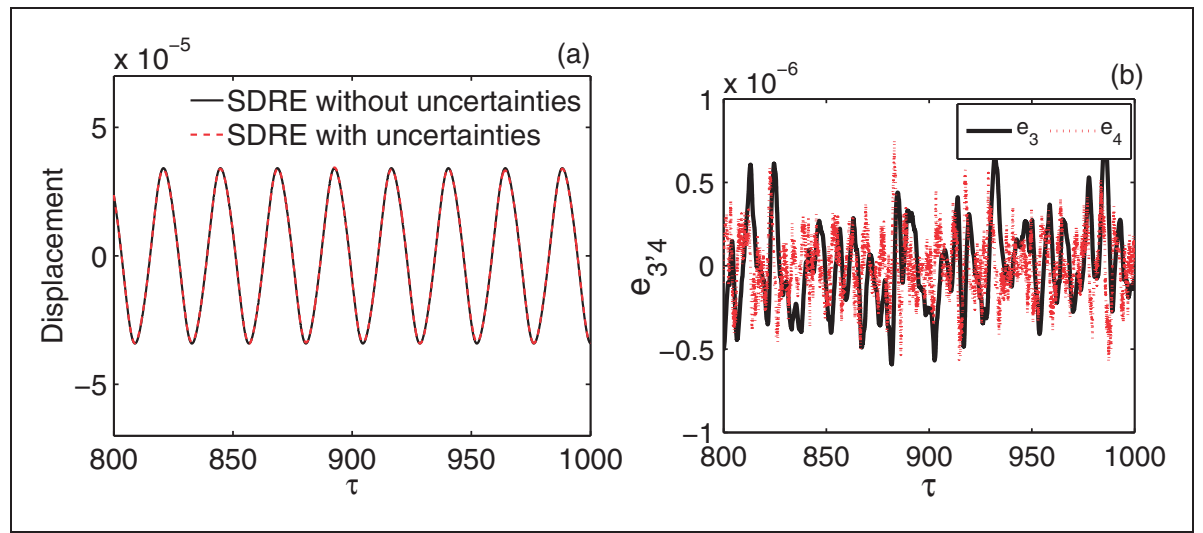

Figure 14. Parameter uncertainties in A, G and $\mathbf{B}$ for $\beta=1.54 \times 10^{8}$ : (a) State-dependent Ricatti Equation (SDRE) control displacement with and without uncertainties; (b) deviation in displacement $\left(e_{3}=x_{3}-\hat{x}_{3}\right)$ and deviation in velocity $\left(e_{4}=x_{4}-\hat{x}_{4}\right)$ error for uncertainty in parameters.

of controller gains $\mathbf{B}$ and in the vector that represents the external perturbation $\mathbf{G}$ will be considered to have a random error of $\pm 20 \%$. This is a strategy similar to that of Nozaki et al. (2013), Tusset et al. (2013, 2015) and Balthazar et al. (2013, 2014a, 2014b).

In Figures 9-11 we observe the robustness of the control to maintain the system in periodic orbit with displacements near to those obtained using SDRE control for uncertainties, when $\beta=7.578 \times 10^{7}$, in $\mathbf{A}, \mathbf{A}$ and $\mathbf{G}$ and $\mathbf{A}, \mathbf{G}$ and $\mathbf{B}$, respectively. $\mathbf{e}=[\mathbf{x}-\hat{\mathbf{x}}]$ represents the trajectory deviation obtained with control without uncertainties $(\mathbf{x})$ and the trajectory obtained with control with parametric uncertainties $(\hat{\mathbf{x}})$, considering the variation of the parameters as follows: $\hat{\delta}=\delta(0.8+0.4 r(\tau)), \quad \hat{\zeta}=\zeta(0.8+0.2 r(\tau)), \quad \hat{\alpha}=\alpha(0.8+$ $0.2 r(\tau)), \quad \hat{\eta}=\eta(0.8+0.2 r(\tau)), \quad \hat{\beta}=\beta(0.8+0.2 r(\tau))$, $\hat{\sigma}=\sigma(0.8+0.2 r(\tau)), \quad \hat{\gamma}=\gamma(0.8+0.2 r(\tau)), \quad \hat{\kappa}=\kappa(0.8+$ $0.2 r(\tau))$ and $\hat{\chi}=\chi(0.8+0.2 r(\tau))$, where $r(\tau)$ are normally distributed random functions.
The robustness for the second attractor, $\beta=1.54 \times 10^{8}$, is shown in Figures $12-14$ for uncertainties in $\mathbf{A}, \mathbf{A}$ and $\mathbf{G}$, and $\mathbf{A}, \mathbf{G}$ and $\mathbf{B}$, respectively.

It is possible to observe that the proposed SDRE control is effective in controlling the chaotic orbit of the nonlinear system, even in the presence of parametric errors and noise, as can be observed in Figures 9(a)14(a). The errors in displacement $\left(e_{3}=x_{3}-\hat{x}_{3}\right)$ and velocity $\left(e_{4}=x_{4}-\hat{x}_{4}\right)$ of the system due to uncertainties have small values, as shown in Figures 9(b)-14(b).

\section{Conclusions}

This paper presented the horizontal dynamic behavior of a vertical transportation system harmonically excited by guide rails, as well as a proposal for a control strategy using the SDRE technique. The nonlinear dynamic system, with a pure cubic Duffing spring representing the influence of the traction cable on the system, was 
analyzed. We observed that the response of the system was highly influenced by the nonlinear term of the Duffing spring. This system presents different qualitative responses, such as periodic or chaotic behavior, depending on the nonlinear Duffing spring parameter used. Using the SDRE control technique described in this work, the chaotic attractor shown in Figures 7 and 8 could be controlled to a periodic orbit even in the presence of parametric errors and noise. It could be established, as a consequence of the obtained results, that there was a decrease of the amplitude of the cabin's motions. In addition, the SDRE control can be used to contribute to the preservation of the elevator component integrity. It is possible to observe that the amplitude of the acceleration was reduced and, therefore, an increase in the passenger comfort level during the motion of the elevator may be detected.

\section{Declaration of Conflicting Interests}

The author(s) declared no potential conflicts of interest with respect to the research, authorship, and/or publication of this article.

\section{Funding}

The author(s) received no financial support for the research, authorship, and/or publication of this article.

\section{References}

Arrasate X, Kaczmarczyk S, Almandoz G, et al. (2014) The modelling, simulation and experimental testing of the dynamics responses of an elevator system (1-2). Mechanical Systems and Signal Processing 42: 258-282.

Balthazar JM, Bassinello DG, Tusset AM, et al. (2014a) Nonlinear control in an electromechanical transducer with chaotic behaviour. Meccanica 49(8): 1859-1867.

Balthazar JM, Tusset AM and Bueno AM (2014b) Nonlinear TM-AFM control considering parametric errors in the control signal evaluation. Journal of Theoretical and Applied Mechanics (Warsaw) 52: 93-106.

Balthazar JM, Tusset AM, Souza SLT, et al. (2013) Microcantilever chaotic motion suppression in tapping mode atomic force microscope. Proceedings of the Institution of Mechanical Engineers, Part C: Journal of Mechanical Engineering Science 227(8): 1730-1741.

Beltrán-Carbajal F and Silva-Navarro G (2014) Active vibration control in Duffing mechanical systems using dynamics vibration absorbers. Journal of Sound and Vibration 333: 3019-3030.

Benosman M (2014) Lyapunov-based control of the sway dynamics for elevator ropes. IEEE Transactions on Control Systems Technology 22(5): 1855-1863.

Carrella A, Brennan MJ, Waters TP, et al. (2012) Force and displacement transmissibility of a nonlinear isolator with high-static-low-dynamic-stiffness. International Journal of Mechanical Sciences 55: 22-29.

Chang CC, Lin CC, Su WC, et al. (2011) H $\infty$ direct output feedback control of high-speed elevator systems. In:
ASME 2011 pressure vessels and piping conference 8: seismic engineering, Baltimore, MD, USA, 17-21 July 2011, pp.289-296.

Chi RM and Shu HT (1991) Longitudinal vibration of a hoist rope coupled with the vertical vibration of an elevator car. Journal of Sound and Vibration 148(1): 154-159.

Çimen T (2010) Systematic and effective design of nonlinear feedback controllers via the State-Dependent Riccati Equation (SDRE) method. Annual Reviews in Control 34(1): 32-51.

Feng Y, Zhang J and Zhao Y (2009) Modeling and robust control of horizontal vibrations for high-speed elevator. Journal of Vibration and Control 15(9): 1375-1396.

Fortune JW (1997) Mega-high-rise elevatoring. Elevator World 45(12): 128-135.

Fu WJ, Liao XB and Zhu CM (2005) Structural optimization to suppress elevator horizontal vibration using virtual prototype. Acta Simulata Systematica Sinica 6: 1500-1504.

Funai K, Katayama H, Higaki JI, et al. (2004) The development of active vibration damper for super high-speed elevators. Lift Report 5: 22-37.

Kaczmarczyk S and Iwankiewicz R (2006) Dynamic response of an elevator car due to stochastic rail excitation. Proceedings of the Estonian Academy of Sciences Physics Mathematics 55(1): 58-67.

Kaczmarczyk S, Iwankiewicz R and Terumichi Y (2009) The dynamic behaviour of a non-stationary elevator compensating rope system under harmonic and stochastic excitations. Journal of Physics: Conference Series 181(012047): 1742-6588.

Kaczmarczyk S and Picton P (2013) The prediction of nonlinear responses and active stiffness control of moving slender continua subjected to dynamic loadings in a vertical host structure. International Journal of Acoustics and Vibration 18(1): 39-44.

Kaplan JL and Yorke JA (1979) Chaotic behavior of multidimensional difference equations. In: Peitgen $\mathrm{HO}$ and Walther HO (eds) Functional Differential Equations and Approximation of Fixed Points 730. Berlin: Springer, pp. 204-227.

Khan F, Stoeber B and Sassani F (2014) Modeling and simulation of linear and nonlinear MEMS scale electromagnetic energy harversters for random vibration environments. The Scientific World Journal 2014: 1-15.

Kovacic I and Brennan MJ (2011) The Duffing Equation: Nonlinear Oscillators and their Behaviour. New Jersey, USA: John Wiley \& Sons.

Lopez SMR, Perondi EA and Sobrinho MRS (2010) Adaptive control for an active suspension of an elevator. ABCM Symposium Series in Mechatronics 4: 62-71.

Meirovitch L (1970) Methods of Analytical Dynamics. New York, USA: McGraw-Hill.

Mitsui N and Nara T (1971) Analysis of horizontal quaking of high-speed elevators. Hitachi Review 20(8): 342-348.

Mracek CP (2007) SDRE autopilot for dual controlled missiles. Automatic Control in Aerospace 17(1): 750-755.

Munakata T, Kohara H, Takai K, et al. (2003) The world's fastest elevator. Elevator World 51(9): 97-101.

Nai K, Forsythe W and Goodall RM (1994) Vibration reduction techniques for high speed passenger elevators, In: 
Proceedings of the third IEEE conference on control applications, Glasgow, Scotland, UK, 23-25 August 2010, pp.965-970.

Nayfeh AH (1973) Perturbation Methods. New York: Wiley.

Noguchi N, Arakawa A, Miyata K, et al. (2011) Study on active vibration control for high-speed elevators. Journal of System Design and Dynamics 5(1): 164-179.

Nozaki R, Balthazar JM, Tusset AM, et al. (2013) Nonlinear control system applied to atomic force microscope including parametric errors. Journal of Control, Automation and Electrical Systems 24(3): 223-231.

Ogata K (2009) Modern Control Engineering, 5th ed. Boston, USA: Prentice Hall.

Oh J, Peng P, Winston H, et al. (2006) Elevator active suspension utilizing repulsive magnetic force. Patent US2006/ 0175150A1, USA.

Pearson JD (1962) Approximation methods in optimal control I. Sub-optimal control. International Journal of Electronics 13(5): 453-469.

Roberts R (1998) Control of high-rise/high-speed elevators. In: Proceedings of the American control conference, 6, Philadelphia, PA, 22-26 June 1998, pp.3440-3444.

Rodrigues KS, Balthazar JM, Tusset AM, et al. (2014) Preventing chaotic motion in tapping mode atomic force microscope. Journal of Control, Automation and Electrical Systems 25(6): 732-740.

Sajeeb R, Manohar CS and Roy D (2007) Use of particle filters in an active control algorithm for noisy nonlinear structural dynamical systems. Journal of Sound and Vibration 306(1): 111-135.

Sandilo SH and van Horssen WT (2015) On a cascade of autoresonances in an elevator cable system. Nonlinear Dynamics 80(3): 1613-1630.

Sharma A, Patidar V, Purohit G, et al. (2012) Effects on the bifurcation and chaos in forced Duffing oscillator due to nonlinear damping. Communications in Nonlinear Science and Numerical Simulation 17(6): 2254-2269.

Singh NM, Dubey J and Laddha G (2008) Control of pendulum on a cart with state dependent Riccati equations. World Academy of Science, Engineering and Technology 2(5): 671-675.

Stansbery DT and Cloutier JR (2000) Position and attitude control of a spacecraft using the state-dependent Riccati equation technique. In: proceedings of the American control conference, Chicago, IL, EUA, June 2000, pp.1867-1871.

Triguero RC, Murugan S, Gallego R, et al. (2013) Robustness of optimal sensor placement under parametric uncertainty. Mechanical Systems and Signal Processing 41(1): 268-287.

Tu JY, Lin PY and Cheng TY (2015) Continuous hysteresis model using Duffing-like equation. Nonlinear Dynamics 80: 1039-1049.

Tusset AM, Balthazar JM, Bassinello DG, et al. (2012a) Statements on chaos control designs, including a fractional order dynamical system, applied to a MEMS comb-drive actuator. Nonlinear Dynamics 69(4): 1837-1857.

Tusset AM, Balthazar JM, Chavarette FR, et al. (2012b) On energy transfer phenomena, in a nonlinear ideal and nonideal essential vibrating systems, coupled to a (MR) magneto-rheological damper. Nonlinear Dynamics 69(4): $1859-1880$.

Tusset AM, Balthazar JM and Felix JLP (2012c) On elimination of chaotic behavior in a non-ideal portal frame structural system, using both passive and active controls. Journal of Vibration and Control 19: 803-813.

Tusset AM, Bueno AM, Nascimento CB, et al. (2013) Nonlinear state estimation and control for chaos suppression in MEMS resonator. Shock and Vibration 20(4): 749-761.

Tusset AM, Piccirillo V, Bueno AM, et al. (2015) Chaos control and sensitivity analysis of a double pendulum arm excited by an RLC circuit based nonlinear shaker. Journal of Vibration and Control 1: 11.

Utsunomiya K, Okamoto K and Yumura T (2004) Guide for elevator. Patent 6,786,304, USA.

Utsunomiya K, Okamoto K and Yumura T (2006) Active horizontal vibration reducing device for elevator. Patent 7,007,774, USA.

Venkatesh SR, Cho YM and Kim J (2002) Robust control of vertical motions in ultra-high rise elevators. Control Engineering Practice 10(2): 121-132.

Wolf A, Swift JB, Swinney HL, et al. (1985) Determining Lyapunov exponents from a time series. Physica 16D: 285-317.

Yang IH, Jeong JE, Jeong UC, et al. (2014) Improvement of noise reduction performance for a high-speed elevator using modified active noise control. Applied Acoustics 79: $58-68$.

Zhang RJ, Yang WW and Wang XW (2014) The reliability analysis of horizontal vibration of elevator based on multistate fuzzy Bayesian network. Jordan Journal of Mechanical and Industrial Engineering 8(1): 43-49.

Zhang XG, Guo KJ, Li HG, et al. (2008) A new friction model for the slide guide in elevator systems: experimental and theoretical investigations. Proceedings of the Institution of Mechanical Engineers, Part C: Journal of Mechanical Engineering Science 222(11): 2177-2189.

Zhu WD and Teppo LJ (2003) Design and analysis of a scaled model of a high-rise, high-speed elevator. Journal of Sound and Vibration 264(3): 707-731.

\section{Appendix}

Notation

A Matrix of state variables $(6 \times 6)$

$a_{0} \quad$ Amplitude of external excitation (m)

B Matrix of controller gains $(6 \times 1)$

$b$ Damping coefficient of the suspension (N.s/m)

c Damping coefficient of the cabin (N.s/m)

$D_{\mathrm{KY}} \quad$ Kaplan-Yorke dimension (dimensionless);

$E_{\text {pel }}$ Potential energy associated to the nonlinear Duffing spring (Nm)

$F_{\text {el }} \quad$ Restitution force associated to the nonlinear Duffing spring $(\mathrm{N})$ 
G Vector that represents external perturbations that do not depend on the states $(6 \times 1)$

$J$ Cost function

Jerk First derivative of acceleration $\left[\mathrm{ms}^{-3}\right]$;

$k_{1} \quad$ Stiffness coefficient of the guide rollers $(\mathrm{N} / \mathrm{m})$

$k_{2}$ Stiffness coefficient of the suspension $(\mathrm{N} / \mathrm{m})$

$k_{3} \quad$ Stiffness coefficient of the elevator ropes referring to tilting motion of the cabin

$k_{3 \mathrm{~L}} \quad$ Linear stiffness coefficient of the elevator ropes referring to tilting motion of the cabin $(\mathrm{N} / \mathrm{m})$

$k_{3 \mathrm{NL}} \quad$ Nonlinear stiffness coefficient of the elevator ropes referring to tilting motion of the cabin $\left(\mathrm{N} / \mathrm{m}^{3}\right)$

$L \quad$ Lagrangian

M Controllability matrix $(6 \times 6)$

$M_{\text {eq }} \quad$ Mass of the cabin $(\mathrm{kg})$

$m_{\text {eq }} \quad$ Mass of the suspension system $(\mathrm{kg})$

P Solution of the Riccati equation $(6 \times 6)$

Q Symmetric positive definite matrix $(6 \times 6)$

$Q_{\mathrm{k}} \quad$ Sum of conservative and nonconservative generalized forces $(\mathrm{N})$

R Positive definite matrix $(1 \times 1)$

$t$ time (s)

$T \quad$ Kinect energy (N.m)

U Control vector $(1 \times 1)$

V Potential energy (N.m)

$\omega_{\mathrm{e}}$ External excitation frequency $(\mathrm{rad} / \mathrm{s})$

$\omega_{0} \quad$ Natural frequency $(\mathrm{rad} / \mathrm{s})$

$X$ Displacement of the cabin (m)

$\mathbf{x}$ State vector for dimensionless model $(6 \times 1)$

$X_{\text {est }} \quad$ Constant used in dimensionless equations (m)

$X_{\mathrm{L}} \quad$ Displacement of the left suspension system (m)
$X_{\mathrm{R}}$ Displacement of the right suspension system (m)

$X_{\mathrm{LB}}$ External excitations caused by guide rails deformations (left) (m)

$X_{\mathrm{RB}}$ External excitations caused by guide rails deformations (right) (m)

$x_{1}$ Displacement of the left-hand suspension system for the dimensionless model

$x_{2} \quad$ First derivative of $\left(x_{1}\right)$

$x_{3}$ Displacement of the cabin for dimensionless model

$x_{4} \quad$ First derivative of $\left(x_{3}\right)$

$x_{5}$ Displacement of the left-hand suspension system for the dimensionless model

$x_{6} \quad$ First derivative of $\left(x_{5}\right)$

$\alpha$ Stiffness coefficient of the suspension

$\beta$ Nonlinear stiffness coefficient of the elevator ropes referring to tilting motion of the cabin

$\gamma$ Amplitude of external excitation

$\delta$ Sum of the stiffness coefficient of the guide rollers and stiffness coefficient of the suspension

$\zeta$ Damping coefficient of the suspension

$\eta \quad$ Stiffness coefficient of the suspension

$\kappa$ Component $b_{2,1}$ of the matrix of controller gains (B)

$\tau \quad$ Time

$\chi$ Component $b_{4,1}$ of the matrix of controller gains (B)

$\Omega \quad$ External excitation frequency 